Published in Philosophy Today, SPEP Supplement 1999, pp 88-98

\title{
The Relevance of Nonsymbolic Cognition \\ to Husserl's Fifth Meditation \\ by
}

Albert A. Johnstone
University of Oregon

\section{Two Objections}

In his $\underline{\text { Cartesian Meditations }}$ at the outset of the Fifth Meditation, Edmund Husserl

proposes to sketch an account of how the meaning, 'other person,' comes to be bestowed on certain items encountered in the perceived surrounding world. As he states the matter, he proposes to "discover in what intentionalities, syntheses, motivations, the sense 'other ego'

becomes fashioned." ${ }^{1}$ An account of the constitution of such a sense is a necessary step in an account of how objects in the world come to have the sense 'there for others'. The latter demonstration is itself, of course, a necessary step in the larger phenomenological project of showing how the perceived world comes to have the sense it has in the natural attitude of everyday adult life.

In introducing his projected account of sense constitution, Husserl presents it as an answer to the general charge that phenomenology entails transcendental solipsism. He judges, and rightly so, that the introduction of the sense 'other' adds a dimension of meaning that converts the perceived world into a common world, with the result that all apparent support is withdrawn from the accusation that phenomenology is transcendental solipsism. Unfortunately, his placing of an allusion to solipsism at the beginning rather than at the conclusion of his account (or better still, in a footnote), has often led to a construal of that account as an attempt to answer the philosophical problem of solipsism. Such an interpretation is clearly mistaken. Sense constitution is an exercise in semantics, and not in ontology. Consequently, the account can 
hardly be intended as a refutation of solipsism, much less a refutation via a demonstration of the existence of other persons.

Admittedly, the situation is somewhat apt to engender confusion. An account of semantic constitution traces out the nature of the evidential situation grounding the constituted sense, and hence closely parallels an epistemological account of knowledge constitution. In addition, Husserl claims in his discussion of skepticism in Ideas I that an appreciation of the evidential situation suffices to reveal "the countersense" committed by those skeptical theses that view essential peculiarities of evidence as deficiencies. ${ }^{2}$ As a result, it is inevitable that the account of semantic constitution should contain, implicitly at least, a rebuttal of certain solipsistic theses, namely those committing a countersense of the sort noted by Husserl. Be this as it may, the solipsistic theses affected could not include the classical solipsistic thesis that questions the existence of other minds, where 'a mind' has the sense of 'a private world of representations'. The reason is simply that the sense, 'private world of representations', can only be a subsequent addition to the sense 'other person' being constituted in Husserl's account, and hence a sense available for solipsistic manoeuvres only on the later occasion of its constitution.

The present paper proposes to answer two sorts of objections commonly raised against the constitution of the sense 'other' sketched in the Fifth Meditation. One sort features the claim that the sense constituted in Husserl's account is not the genuine sense of 'other subject'--that the proper sense includes essential dimensions ignored in the account, dimensions such as the person's social status and function, ${ }^{3}$ or the person's radical irreducibility to another person's possible acts of consciousness. ${ }^{4}$ The second sort of objection centers on the claim that the proposed derivation is circular, that is, that it must presuppose what it sets out to constitute. Here some elaboration is in order. 
Objections of the second sort readily grant that Husserl is right in his estimation of the nature of the task, that to avoid circularity the projected constitution of the sense, 'other subject' must begin at a semantic level that contains no trace of that sense--a level Husserl terms "the sphere of ownness." They grant too that Husserl is right to propose the execution of "a peculiar kind of epoché," ${ }^{5}$ one in which abstraction is made from all intentionalities relating in any way to other subjects. They take him to be mistaken only in thinking that such an abstraction may be carried out. Objections diverge somewhat as to the reasons for deeming the requisite semantic level of abstraction impossible of attainment. According to one currently popular view, we acquire our concepts on acquiring the public medium that is language, a claim often presented in the form of the slogan, "We are born into language." ${ }^{6}$ According to another line of thought, we are irreducibly social animals, so much so that the sense 'other' permeates in one form or another all our concepts. On yet another widespread line of thinking, views of the world vary radically from culture to culture, a fact that shows our very concepts to be culturally relative, hence culturally constructed, and consequently imbued with the sense 'other' in some form.

Now, the two above-noted sorts of objections are based on misunderstandings as to the nature of concepts. Objections of the first sort, those maintaining the inadequacy of the constituted concept, fail to recognize that concepts are complex and built up over time. As a result, what is proposed as the genuine concept of 'other subject' is not seen for what it is in fact: a further elaboration of the concept, one reached only at a much later stage of development. Objections of the second sort overlook the existence of nonlinguistic, noncultural concepts. They fail to recognize the presence of such concepts at the core of linguistic concepts, where they serve as the necessary foundation on which the latter are built. In addition, they fail to recognize the origin of such concepts in the individual's own cognitive endeavors, endeavors that society or 
culture may channel, promote, or stifle to a considerable extent, but never create. What follows is a preliminary sketch aimed towards a substantiation of these various claims.

\section{Nonsymbolic Concepts}

Let us begin with the matter of the existence of nonlinguistic concepts, or to simplify discussion, nonsymbolic concepts, that is, concepts for which the carriers of meaning are neither words spoken or written, nor images of any sort (visual, auditory, or olfactory) but simply what is actually perceived in perceptual encounters. The existence of such concepts is strongly suggested by the intelligent behavior of creatures quite devoid of language, creatures such as animals and infants of every stripe. Very often in the study of adult human cognition, this fact is politely acknowledged, then treated as irrelevant to subsequent investigation. Such an attitude is fostered perhaps by the odd tendency among philosophers to value the disciplines of sociology and anthropology over those of child psychology and primatology. Yet, it generates an account of cognition that is adultist and inadequate. In view of the evolutionary continuity between other primates and humans, and of the developmental continuity between infants and adults, it would be utterly astounding if language acquisition had the effect of effacing totally in adult humans any residue of what are clearly adaptationally successful aptitudes. On the contrary, it is rather to be expected that the more powerful tool of language should build upon and supplement the preexisting cognitive practices, allowing their exercise to remain quite widespread in everyday adult experience.

Let us turn, then, to adult human experience, which is where phenomenology would begin in any event. A close survey of one's own adult experience readily finds a wealth of instances of nonsymbolic cognition that are quite independent of language. Let us consider briefly a few such instances drawn from four domains of perceptual cognition: bodily awareness, 
recognition, awareness of location, perception of objects.

(i) Awareness of one's own body.

Let us suppose that while reading, I am vaguely aware of a persistent itch, and I scratch the back of my head, which is to say, I raise and flex my left arm, touch close to the place of the itch with bent fingers, then initiate a scratching movement that carries my nails across the spot. No language is involved in the operation. I neither declare my intentions to anyone, nor give myself instructions as to how to proceed. I did not learn to scratch my head through following instructions, whether those of a tutor or those in an owner's manual. Indeed, no instructions could state with any degree of accuracy what peculiar combination of tensions and flexings is necessary in order to raise my arm and bring my hand to the appropriate location, and, despite my proficiency in the activity, I would be quite incapable of articulating a detailed recipe for success. In bodily movements of the sort, language is both inadequate and superfluous.

A somewhat analogous situation holds with regard to felt events in one's body. The services of language are irrelevant to knowing what the experience of hunger is like, what it is like to feel contented, or irritable, curious or listless, what to expect of imminently impending events such as sneezes, spasms, outbursts of frustration, and waves of delight, what affective stance to expect to be elicited by visual or auditory irruptions of particular sorts into one's surrounds. An awareness of one's own bodily affective dynamics, at least to some minimal degree, is made unavoidable by the mere fact of being conscious. One's body is always present in one's awareness, marginally or focally, as a highly complex, and often volatile array of tensions, buoyancies, inner contractions, irritations, and insipidities. Linguistic articulation is clearly not required for there to be such awareness. Furthermore, for the most part when language does enter the scene, it is unconcerned with capturing anything but certain quite gross features of what is a 
rich and complex whole.

(ii) Perceptual recognition.

Consider a simple case, my recognizing my friend George in a sea of faces. If asked, I would be quite incapable of explaining how I recognize him. I might tentatively mention George's curly hair, but without feeling the explanation was adequate. On further reflection I might speak of his long face, his sad blue eyes, and pale complexion, but only do so on reflection and with the uncomfortable feeling that the description is too general to get to the heart of the matter. For instance, George's face is not simply long; it is quite long comparatively, oval at the bottom with a sharp protruding chin, while the hairline is somewhat hidden by short curls, and the ears, although protruding, are set back somewhat from the line of the face. I could continue thus giving more and more precise descriptions, each well after the fact and each inadequate to capture the specific shape, proportions, and coloration of the various facial features in virtue of which I instantly recognize George. Since I find it so difficult to capture linguistically exactly what it is I recognize, it must be the case that I recognize independently of the services of language--that I have a nonlinguistic concept of George.

The situation is similar in a vast number of cases, in recognizing items such as the neighbor's cat, the kitchen, one's car in a parking lot, one's coat in a coat room, one's favorite spot where the fishing is good. The item in question is recognized automatically, as it were, despite one's inability to formulate satisfactorily in language the unique familiar features that make the individual different from others. The recognition is physiognomic and fine-grained, quite different in nature from checking through a list of linguistically conceptualized general features of the sort used, for instance, in identifying an expected but unknown passenger at an airport. ${ }^{7}$ It seizes upon a certain peculiar constellation of features--shapes, proportions, colorations, kinetic 
dynamics, that form a whole that is not analyzable with the words, and hence the concepts made available by one's language.

Physiognomic recognition of the sort is also often involved in the recognition of natural kinds. It is not uncommon to find that no adequate description can be given of the nature of the specific features decisive in identifying members of a natural kind, features such as the characteristic square shape of a cow, its wide-muzzled convex nose-bridge, its heavy, awkward gait, or features such as the characteristic sheen, texture, and hues of slate, or copper. Recognition in such cases means recognizing features that can be only roughly and inadequately characterized in the categories made available by language. The features are best conveyed to another speaker nonlinguistically by putting a sample of the relevant kind at the person's disposal for observation.

The existence of such cases of recognition, whether of individuals or of natural kinds, cases involving recognition through features for which language has no name, hence no concept, of course implies that there exist nonlinguistic concepts--where having such concepts simply means being able to recognize perceptually with no assist from language.

(iii) Awareness of one's spatial location.

I hurry home through familiar streets, here turning a corner, there crossing a square, periodically scanning my surrounds while my thoughts flit erratically about. I know where I am going and how to get there. At any point on my way home, I simply recognize my surrounds, and know in what direction to proceed next. I have no need of verbal instructions or outline, any more than a map or an imagistic rehearsal of the experience. Indeed, I may well be quite incapable of giving myself accurate verbal instructions, of pointing out, for instance, that the fountain on the right has a lion's head, or that the next turn is the third street on the left, or that 
there is a bakery on the opposite corner. The situation is similar to that in the hearing of a familiar song, where in the presence of any given line, I simply know what comes next. I have a concept of my surrounds in the sense that I recognize certain places as familiar, have some awareness of what may be found there, and expect to encounter certain further places if I should proceed in particular directions. As one might put the matter unhelpfully, I have a nonsymbolic cognitive map.

(iv) Perception of material objects.

Let us suppose that, feeling moderately peckish, I notice some fruit in a bowl, grasp a shiny bright apple, and bite into it. I expect the apple to have features falling within a certain range of variation, a certain weight, solidity, taste, texture, aroma, crispness. Without giving any thought to the matter, I assume that the apple will be less heavy than a cannon-ball, will not disintegrate in my grasp, will not taste of garlic, and I would be astounded to find otherwise. My expectations can hardly be attributed to the services of language. Language is unnecessary to effectuate the association of the various noticeable co-present features of the apple, just as it is powerless to generate expectations of the taste, texture, and fragrance peculiar to apples.

Likewise, the concept of an individual material object, as applied to unattached, graspable, and easily moved everyday objects, or as applied to animals that move about, cannot plausibly be viewed as a creation of language. To be an individual in such cases is simply to be detached and displaceable and largely the same over time while being only partially perceived at any given time. Awareness of the existence of such entities is primarily a matter of putting successive experiences of an individual together in some way, and would seem quite within the capacity of nonsymbolic cognition lacking any assist from language. Indeed, if a subject had no such capacity, and was utterly devoid of any such awareness, it is quite unclear how language 
could possibly replace the missing capacity, or effectively communicate the awareness.

The above examples of nonsymbolic concepts operative in intelligent goal-directed activity clearly might be multiplied and expanded to other domains. Just as clearly, they counter the claim that all concepts operative in adult life are impregnated with language. Present day thought often considers it a truism that language supplies the categories of thought, creating classifications built on arbitrary divisions within a multidimensional continuum. The truth is quite different. No assist is needed from language to recognize such distinctive qualitative phenomena as the flavor of banana, the smell of methane or of wet dog, the call of a gull, the stealthy flow of feline movement. The phenomena are sufficiently striking and distinctive in their own right to be noticed and subsequently recognized as familiar. What is true in these more striking cases cannot be plausibly denied in the less striking ones. The claim that language is indispensable for the noticing and subsequent recognition of specific hues, textures, odors, or dynamics, is a quite gratuitous one with no visible means of support. ${ }^{8}$

The above examples further suggest that linguistic concepts themselves are often not the pure creations of language. Linguistic concepts that involve perceptual acquaintance are more often complex constructions built on the prior acquisitions of nonsymbolic cognition, which are appropriated unaltered and put to service. For instance, for speakers acquainted with live bananas, the expectation that soft creamy flesh of a specific fragrance lies within the shiny, yellow skin of a peculiarly shaped object constitutes the core of the linguistic concept of 'banana'. To this nonlinguistic conceptual core, linguistic commerce adds a classificatory structure (e. g. tropical fruit) together with a wealth of second-hand information regarding maturation, arboreal provenance, native environment, the aromatic secret of n-butyl acetate. Information of the sort gleaned in classrooms, kitchens, and lecture halls, from story books, 
pulpits, and old wives' tales, information that molds, enriches and fleshes out the concept, waits ever ready to be brought to awareness through perceptual and linguistic associations. Neither the imposed linguistic classificatory structure nor any of the appended second-hand knowledge alters in any way the nonlinguistic core of the concept, such as the expected aromatic softness of the specific sort peculiar to bananas.

One's concepts of acquaintances--friends and neighbors--are most often built up in similar fashion. At their core is a recognizable and quasi-unique physiognomy, around which clusters a vast array of perceptual and linguistically garnered information. It seems no exaggeration to say that language is a gift transmittable only to creatures who already make sense of the world. ${ }^{9}$

\section{Socio-Cultural Influence}

From the above considerations it is fair to conclude that language can provide no sound grounds for the claim that all our concepts involve the sense 'other person' (and hence none for the claim that Husserl's account of constitution is circular). We have yet to consider the view that socio-cultural indoctrination achieves the envisaged result, that of somehow infusing all our concepts with the sense 'other'. Presumably in this task it is assisted by language, while having additional powerful resources at its disposition. As a result the conceptual situation may be expected to be considerably more complex than it is in the case of language alone. Nevertheless, there are very good reasons for thinking that in their main lines the two situations fail to differ significantly.

Consider the instances of nonsymbolic cognition reviewed earlier. In most cases the recognitions and expectations would seem to occur and to be capable of occurring independently of any socio-cultural contribution. Certainly, on the face of things no socio-cultural assistance 
seems required or even possible for learning such matters as how to get one's arms and legs to move, what encounters to expect with other regions of one's body, how to find a finger that hurts, what to expect next when a laugh is felt to be coming, how to recognize one's mother, how to recognize one's room, what taste to expect milk to have, how to focus one's eyes, how to grasp something, or how to gain awareness that there exist such things as three-dimensional, moveable objects. These various rudimentary cognitive performances are rather the fruit of personal experience and initiative, of a subject's own encounters, discoveries, and observations, in which the participation of others is superfluous in principle at least.

This said, it is undeniable that even at the more rudimentary levels that are our present concern, social conditioning has a substantial influence on one's awareness of the world. One may be encouraged by the social surrounds to pay attention to certain things and to ignore or avoid others, to develop certain cognitive pursuits and abilities and to neglect others. In addition, social grooming may have a profound effect on affective evaluations, one the exact extent of which is difficult to assess. For instance, it is not absurd to imagine being taught nonsymbolically from early childhood to react affectively to liver in any of a variety of socially appropriate ways: to find it delicious, disgusting, awe-inspiring, disturbing, comical, or courageenhancing. Equally great variation may be found in the socially promoted affective attitudes deemed appropriate towards other items in the world, for instance, other groups of humans differing in specific ways.

Nevertheless, it would be a mistake to lay too much emphasis on the import of social grooming, or worse still, to view such grooming as the capricious author of invariably different human perceptions of the world. Even on the supposition of the complete malleability of affective attitudes, it would in no way follow that an analogous situation obtained for core 
concepts of the sort considered earlier. Sensuous characteristics remain constant through the fluctuations in valuational and ideational constructs. Liver, for instance, is recognized or identified as liver, not in virtue of the affective evaluations it elicits, but in spite of them; it is recognized through its sensuous characteristics, its opaque, ferrous coloring, semi-gelatinous consistency, sanguineous aroma, and pungent, cloying bitterness. Social grooming does not and cannot alter sensuous characteristics of the sort.

Admittedly, the focus of interest may vary considerably from culture to culture (as from individual to individual). One culture may encourage perceptual focus on overall relations, another on the characteristics of individual objects. Likewise, greater importance may be accorded aesthetic value rather than the economic possibilities afforded. It does not follow that the members of different cultures may be rightly said to live in different worlds, except of course in a figurative sense. They are more accurately said to seize upon or appreciate different aspects of world. In this context it is also pertinent to recall that many aspects of the world are the object of common or cross-cultural appreciation. In all cultures, people have fingers, wind blows hair, and water makes things damp.

As regards the interests themselves, the variability of human affectivity with culture is obviously too vast a topic for minimally adequate treatment here. Yet, a curious fact might be noted, one that emerges persistently from cross-cultural studies. It is that the various human affective propensities are common to all cultures, proclivities to curiosity, delight, discomfort, surprise, frustration, apprehension, grief, playfulness, rage, aesthetic appreciation. It can hardly be the case that the affective propensities themselves are created by particular cultures. Rather they are merely channeled, awakened or repressed by social incentives. Indeed, the commonality of affective propensities suggests further that these abilities are natural, and as such might be 
exercised in principle independently of social encouragement or constraints. The latter suggestion gains considerable corroboration from the findings of a cursory phenomenological examination of affective experience. Whereas the cognitive constituent in such experience is a function of prior experience, in particular, social experience, the affective element proper is a felt sensuous process within one's felt body. In this regard it is akin to the sensuous features of one's surrounds, and as such, is not plausibly considered a social creation.

\section{The Rationality of Nonsymbolic Cognition}

One source of the disparaging treatment often accorded nonsymbolic concepts is the fact that they are largely the passively generated progeny of natural processes. Accordingly, they are deemed to be instinctual and to lack any claim to rationality, a property attributable exclusively, on this line of thinking, to human reasoning operating in the medium of language. Rationality is a rule-governed game played only in community settings, one that descends, as Richard Rorty puts it, "on the shoulders of the bright child somewhere around the age of four, without having existed in even the most primitive form hitherto." ${ }^{10}$ The truth of the matter is in fact quite different: not only is nonsymbolic cognition rational, but it provides the pancultural foundations without which no linguistically articulated rationality could arise. A brief defense of the point will not be amiss.

Consider the operations habitually present in nonsymbolic cognition. By far the most common is expectation of phenomena of a particular type--synchronic in the expectation of contemporaneous phenomena, diachronic in the expectation of future ones. Since expectations of the sort arise from past experience of sequences or concomitances of like types, they are based on evidence, not on a gratuitous hunch, and so are hardly irrational. Furthermore, the expectations often have a degree of confidence that is a direct function of the experienced 
number of confirming instances of events of the type. For this reason they may plausibly be considered the fruit of a rudimentary form of inductive generalization, and indeed, as Husserl suggests, the original or basic form of induction. ${ }^{11}$ Further still, they are often based on observations that are quasi-random in that they are gleaned in the free play of one's spontaneous activity, and hence arise from a more sophisticated and rationally satisfying form of induction. Spontaneous activity makes for quasi-random observations, observations that in a loose sense might not have been made, or might have been replaced by observations made at other times. The presence of an observed regularity in such observations makes plausible the presence of the regularity at times of non-observation, so that an expectation of the regularity is actually the most reasonable one to have, given the evidence. ${ }^{12}$

Expectations of the above sorts clearly do not require the services of language. On the contrary, they are a necessary pre-condition for the acquisition of language. Without the natural propensity to expect repetitions of type sequences and concomitances, the pairing of specific sorts of verbal expressions with specific sorts of situations in the world could not occur, and language learning could not even begin.

While nonsymbolic cognition does not possess the means of formulating generalizations, nevertheless it commonly treats actually perceived items as items of a type, and thus as it were, generalizes in situ. The perceptual expectation arising from such automatic induction might from another perspective be considered a telescoped version of the deductive reasoning pattern of Modus Ponens. A common form of Modus Ponens may be represented schematically as follows, with $\mathrm{A}$ and $\mathrm{B}$ each standing for types of phenomena: an $\mathrm{A}$ implies a B; this is an $\mathrm{A}$; therefore, a B is to be expected. Now, in nonsymbolic cognition when the experience of an A gives rise to the expectation of a B, the experience of an A may plausibly be said to encapsulate the two 
premisses, that of there being an A, and that of A's implying B's, since the A is perceived not only as an A, but also as a type implying another type, B. The conclusion is of course present in the form of an expectation of a B.

Nonsymbolic cognition also contains what might be viewed as a collapsed version of Modus Tollens. The latter is a form of reasoning whereby from the fact that an A implies a B, together with the fact there is no B, it is concluded that there is no A. In cases in which the A's and the B's are synchronic, a subject who is aware that A's and B's occur together, may, on seeing no B, expect there to be no A. (For instance, a dog, knowing that the master and his car always arrive together, might presumably, on seeing no car, expect to find no master.) In cases in which the A's and B's are diachronic (the A's preceding the B's), nonsymbolic cognition alone is unable to draw the relevant conclusion. The reason is that in nonsymbolic cognition, since there are no symbols, a conclusion cannot be represented symbolically, and so must take the form of an expectation. Obviously, it is not possible to expect a past event to occur; only present or future phenomena may be expected. Consequently, while nonsymbolic cognition contains what might be considered a concrete form of synchronic Modus Tollens, it cannot, as matters stand, execute the particular form of reasoning, diachronic Modus Tollens. An appeal to imagination to provide an imagistic representation of the absence of an A could, of course, remedy matters.

Nonsymbolic cognition plays a more obvious role in classical logic with its entailments and syllogisms that derive their validity from elementary relations of inclusion in, and exclusion from, groups. Such relations can readily be illustrated or perceived to hold in concrete cases. Admittedly, nonsymbolic cognition can deal with syllogisms involving all members of a group only in cases where the whole of the group is perceived; nevertheless it operates quite effectively within this limitation. Actual cases of syllogistic reasoning in everyday situations are of course 
rather rare, except within Logic classes, but in this latter context, nonsymbolic cognition may be called upon to provide an invaluable service. Groups of individuals or closed figures may be used to illustrate simpler truths of the sort: if all of one group is contained in a second group, then none of that group is outside the second group. Likewise, a valid syllogism itself may be illustrated, for instance, one with premisses that a first group is contained in a second group, and the second group is contained in a third group, and the conclusion that the first group must be contained in the third group. Language is employed in such cases to communicate the truth to be illustrated, or the premisses and conclusion of the argument, the validity of which is to be illustrated. However, grasping the truth or the validity is a matter of looking at the illustrative situation, and seeing that matters could not be otherwise. ${ }^{13}$ It is a matter of seeing there is no possible way in which the truth could be false--no way in which a group contained in another may fail not to be outside the containing group. Or again, it is a matter of seeing there is no possible way in which the syllogism could be invalid, i. e., have its premisses true and its conclusion false--no way for a group to be contained in a second group contained in a third, and yet itself fail to be contained in the third group. Grasping facts of the sort is not a linguistic operation. It is a matter of trying out different possibilities and coming to see in each case that given certain constraints, certain other relations cannot not hold. Such seeing is the work of nonsymbolic cognition--often facilitated by an appeal to the services of imagination. ${ }^{14}$

The situation is similar for fundamental mathematical truths: understanding them is seeing that they could not be otherwise. The more rudimentary of these truths do not require the services of language for their enunciation. When the number of individuals in a group is small, that number may be recognized physiognomically, that is, by its appearance, without it being reached by counting and without it having a name (such as "three" or "five"). In the presence of a 
small group of the sort, elementary truths of addition may be illustrated; for instance, a group of five may be seen as a group of three together with a group of two, or a group of two together with a group of three; both are simply different ways of considering one and the same group of five items. Such seeing amounts to grasping certain arithmetic truths of addition. Elementary truths of multiplication may also be grasped, as, for instance, when two groups of three, or three groups of two are seen as rearrangements of a group of six individuals. In like fashion, without assist from language, simple operations with fractions may be performed, three items seen as six halves, for example. Even in more complicated operations requiring language for their enunciation, understanding depends upon seeing a relationship, and thus requires nonsymbolic cognition. Understanding why division by two fifths is a matter of multiplying by five and dividing by two, can be achieved through manipulations of concrete items such as pies, oranges, or coins.

In all this, language is useful for pointing out similarities and for reinforcing memory. More importantly, it is necessary for generalizing, for dealing with numbers too great to be recognized perceptually, and for making operations become rote: for instance, for being in a position to say, without working the matter out each time, that six times seven is forty-two. Yet, while it is true there could not be very extensive use of arithmetic without language, nevertheless, the grasping of the basic truths of arithmetic is the work of nonsymbolic cognition.

It would seem safe to conclude that illogicality is not an obvious defect of nonsymbolic cognition and of the concepts it generates. Not only do logical operations exist already at the level of nonsymbolic cognition independently of language, but more than this, nonsymbolic cognition with its expected sequences and concomitances, and with its grasping of group and spatial operations, serves as the indispensable foundation for subsequent linguistic reasoning and 
understanding. Socio-cultural pressures may well encourage or demand rational thought and mathematical understanding, but both are already present in nonsymbolic cognition operating independently of social influence--what might be termed 'natural nonsymbolic cognition'.

\section{Conclusions}

The above considerations point overwhelmingly to the conclusion that both linguistically structured cognition and cultural grooming take as their necessary point of departure the operations and findings of natural nonsymbolic cognition. Husserl's proposal to abstract from all intentionalities relating to other subjects must be deemed both reasonable and feasible, and by the same token the charge of circularity against his account of the constitution of the sense 'other person' must be judged to be unwarranted.

The above considerations also strongly suggest that linguistic and culturally formed concepts relating to perceptually encountered items have at their core nonsymbolic, natural concepts. A concept such as that of 'another subject' may reasonably be expected to have a similar structure and formation, that is, to have its origin and core in a nonsymbolic, natural concept that by successive accretions of sense grows into the complex, sophisticated, linguistically structured, culturally and socio-economically groomed concept possessed by the normal adult.

The most rudimentary forms of the sense, 'other self' figure in the sort of experience Husserl discusses in his Fifth Meditation, an experience involving an analogical transfer of sense to an encountered human being. As Husserl points out, the sense-constitution involves two animate organisms, the constituting subject's own animate organism as it is experienced tactually and kinesthetically, and the visually present animate organism of the other person. ${ }^{15}$ The analogical transfer of sense presupposes the prior experience of oneself as a spontaneously 
moving, feeling body, a body of a peculiar shape, structure, and dynamics, both infused with and animated by various affective transformations: discomfort, yearning, enthusiasm, surprise, curiosity. Without a prior nonsymbolic familiarity with oneself, with one's own felt dynamics and affective life, the perception of the analogous structure and dynamics of a perceived body as familiar would be impossible. The analogy in structure is a matter of topological and dynamic congruences between two bodily forms, a topological congruence between the tactual and visual geometrical arrangements and movements, for instance, of two eyes above a mouth and tongue, and a congruence between the tactual and visual dynamics and spontaneity of affective animate behavior such as surprise, agitation, excitement, distress, tenderness. Thus, at the most primitive level, the analogizing transfer of sense consists of the experienced familiarity of the form and movements of a perceptually present animated body, the nonsymbolic fleshing out of the perceived form and movement with an anticipated immediate future, and the endowment of displays of affection, interest, annoyance, or curiosity with an affective halo that readily elicits an affective echo in the experiencing subject. An item in the environment is perceived to be an animate being.

Subsequently, more complex senses may be constituted, senses of the other as perceiving from a different location, the other as thinking, as knowing, or not knowing. At higher levels still may be found the more sophisticated senses Husserl is faulted for not mentioning, the sense of the other as immersed in a network of socio-economic relations, or the sense of the other as radically inaccessible to another person's possible acts of consciousness. The concept of 'another person' is not something static and unchanging, amenable to encapsulation in a dictionary definition; it is something that grows richer over time like most concepts. It is "an open, ever-tobe-corrected concept," to use Husserl's phrase ${ }^{16}$, and one interacting incessantly with the concept 
of 'self' in a process of mutual enrichment. The objection that Husserl's account of constitution in the Fifth Meditation fails to yield the genuine concept of 'other', is one that is due, as is the second objection, to a lack of understanding of the nature of concepts. 


\section{END NOTES}

1. Edmund Husserl, Cartesian Meditations: an Introduction to Phenomenology, tr. Dorion Cairns (The Hague: Martinus Nijhoff, 1973), p. 90.

2. Edmund Husserl, Ideas Pertaining to a Pure Phenomenology and to a Phenomenological Philosophy, First Book, tr. F. Kersten (The Hague: Martinus Nijhoff, 1982), p. 187.

3. See John Sallis, "On the Limitation of Transcendental Reflection, or, Is Intersubjectivity Transcendental?", The Monist pp. 312-333, p, 326.

4. See Peter Hutcheson, "The Primacy of Intersubjectivity," The Modern Schoolman Vol. LIX, number 4, May 1982, pp. 281-5.

5. Husserl, Cartesian Meditations, p. 93.

6. The dictum is enunciated by Lacan, for whom it has in fact a less literal meaning. See Jacques Lacan, The Four Fundamental Concepts of Psycho-Analysis, ed. Jacques-Alain Miller, tr. Alan Sheridan (New York: W. W. Norton, 1978), p. 131. The doctrine itself is widespread, and is implied by many remarks in Wittgenstein's later writings. See, for instance, Ludwig Wittgenstein, Philosophical Investigations, tr. G. E. M. Anscombe (Oxford: Basil Blackwell, 1963), pars. 342, 378, 384.

7.. A similar list is used by the patient in Oliver Sacks, The Man Who Mistook His Wife for a Hat (New York: Harper \& Row, 1985), p. 13.

8. Wittgenstein's laconic claim that we learn the meaning of the concept 'pain' when we learn the language, must be judged a serious misrepresentation of the way language acquisition actually works. See Ludwig Wittgenstein, Philosophical Investigations tr. G. E. M. Anscombe (Oxford: Basil Blackwell, 1963), p. 118.

9. It is perhaps of some interest that the above claims based on phenomenological or introspective findings fit well with the conclusions certain researchers draw from findings in studies of patients with brain lesions. Edoardo Bisiach, for one, makes the following claim: "This suggests that language per se cannot be considered an autonomous form of representation, in the sense that it has no independent data base of its own: all representation (originally) missing in the analogue mode is (derivatively) missing in the verbal mode as well." Edoardo Bisiach, "Language without Thought," pp. 464-84, in L. Weiskrantz (ed.), Thought without Language (Oxford: Clarendon Press, 1988), p. 466.

10. Richard Rorty, Philosophy and the Mirror of Nature (Princeton, N.J.: Princeton University Press, 1979), p. 187.

11.. See Edmund Husserl, Experience and Judgment, ed. Ludwig Landgrebe, trans. James S. Churchill and Karl Ameriks (Evanston, Il: Northwestern University Press, 1973), p. 33.

12. The point is developed further in Albert A. Johnstone, Rationalized Epistemology: Taking Solipsism Seriously (Albany, NY: State University of New York Press, 1991), pp. 289-96.

13. On the assumption that shifts in the focus of attention do not change structures in the world. 14. Interestingly, Husserl speaks of "the originally intuitive life which creates its originally selfevident structures through activities on the basis of sense-experience," and its all too ready 
seduction by language. Edmund Husserl, "Appendix VI: The Origin of Geometry," The Crisis of European Sciences and Transcendental Phenomenology, tr. David Carr (Evanston, IL:

Northwestern U. P., 1970), p. 362.

15. Husserl, Cartesian Meditations, pp. 97, 112.

16. Husserl, Experience and Judgment, p. 333. 